\title{
Familia Chironomidae (Orden Díptera) utilizada como bioindicador para la determinación de calidad ambiental de la cuenca del Río Gallegos (Santa Cruz, Argentina).
}

\section{Family Chironomidae (Order Diptera) used as a bioindicator for the determination of the environmental quality of the Río Gallegos basin (Santa Cruz, Argentina).}

\author{
Carolina L. Sierpe, Alejandro Sunico \\ clsierpe@gmail.com; alejandrosunico@yahoo.com.mx \\ Universidad Nacional de la Patagonia Austral - Unidad Académica Río Gallegos - \\ Departamento de Ciencias Exactas y Naturales. \\ Río Gallegos - Santa Cruz - Argentina
}

Recibido: 15/03/2019. Aceptado: 05/07/2019

\section{RESUMEN}

Estudios previos realizados en la región, utilizando la comunidad de macroinvertebrados bentónicos y aplicando índices bióticos a nivel taxonómico de órdenes y familias, permitieron determinar la existencia de procesos de impacto antrópico y degradación en los ambientes acuáticos de la cuenca. Estos estudios, pusieron en evidencia la necesidad de trabajar a niveles taxonómicos más precisos, abarcando la identificación a nivel de género y especie de algunos de los organismos predominantes en la comunidad bentónica. En función de esto, uno de los objetivos de la presente beca es caracterizar la fauna de insectos quironómidos (Díptera, Chironomidae) para la estación otoño, empleando las técnicas aprendidas para la determinación taxonómica a nivel de género. La información obtenida permitirá ajustar y mejorar la eficiencia de los índices bióticos de calidad ambiental, diseñados y adaptados para el diagnóstico y monitoreo en ambientes acuáticos de la región.

En este trabajo se procesaron 28 muestras de macroinvertebrados bentónicos de 7 sitios de muestreo localizados sobre la cuenca superior del río Gallegos. Estas muestras se tomaron en mayo de 2017 y corresponden a la estación de otoño. Se identificaron un total de 10 géneros de insectos quironómidos, de los cuales 5 pertenecen a la subfamilia Orthocladiinae, 2 de Tanypodinae y 3 de Chironominae.

Palabras clave: familia Chironomidae; macroinvertebrados bentónicos; río Gallegos.

\begin{abstract}
Previous studies realized carried out in the region, using the community of benthic macroinvertebrates and applying bioticindexes at taxonomic level of orders and families, allowed to determine the existence of processes of anthropic impact and degradationin the water environments of the watershed. These studies showed the need to work at more precise taxonomic levels, covering the identification at the level ofgender and species of some of the predominant organismsin the benthic community. Depending on this, one of the objectives of this fellowship is to characterize the fauna of chironomids insects (Diptera, Chironomidae) for the autumn season, using the techniques learned for taxonomic determination at the gender level. The information obtained will allow to adjust and improve the efficiency of the biotic
\end{abstract}


índices of environmental quality, designed and adapted for the diagnosis and monitoring in aquatic environments of the region.

In this paper, 28 samples of benthic macro invertebrates were processed from 7 sampling sites located on the upper Río Gallegos basin. These samples were taken in May 2017 and correspond to the autumn season. A total of 10 genera of chironomids insects were identified, of which 5 belong to the subfamily Orthocladiinae, 2 of Tanypodinae and 3 of Chironominae.

Key Words: Chironomidae family; benthic macro-invertebrates; Río Gallegos.

\section{INTRODUCCIÓN}

La comunidad del macrobentos está compuesta por organismos que viven en estrecha vinculación con el fondo de los ambientes acuáticos y, por lo tanto, en relación intrínseca con el sustrato y con las sustancias tóxicas que se encuentran en él (Hellawell 1986, AlbaTercedor 1996, Pave y Marchese 2005). Los organismos que integran el macrobentos, son aquellos que pueden ser retenidos por una malla de luz de $500 \mu \mathrm{m}$ y tienen un tamaño que les permite ser percibidos a simple vista, lo cual facilita mucho su recolección y su estudio. En el agua dulce, se encuentran representados por numerosos filos animales, tales como anélidos, moluscos, platelmintos, nematodos y artrópodos (principalmente insectos). Entre los artrópodos, los órdenes más representados en los sistemas lóticos de Patagonia corresponden a los insectos Ephemeroptera, Plecoptera, Trichoptera, Díptera y Coleóptera, y a los crustáceos Amphipoda (Miserendino 2001, Domínguez y Fernández 2009). Algunos de estos grupos pasan todo, o casi todo, su ciclo de vida en el agua (Hanson et al. 2010).

Diversos autores afirman que las comunidades de macroinvertebrados bentónicos, reflejan la calidad de los ecosistemas acuáticos en los cuales habitan, es por ello, que los métodos de evaluación ambiental basados en el estudio de estas comunidades han sido ampliamente utilizados desde hace varias décadas en todo el mundo (Alba-Tercedor 1996, Prat et al. 2009, Mauad 2014, Crettaz-Minaglia et al. 2014, Roldán-Pérez 2016, Torrentes et al. 2016).

Las técnicas que utilizan macroinvertebrados acuáticos como indicadores de contaminación ambiental, han demostrado su total eficacia en la detección de fuentes de alteración antrópica (Bonada et al. 2006) y en el cartografiado de la calidad de las aguas (Alba-Tercedor 1996). La abundancia y la proporción en la comunidad de especies sensibles y tolerantes a la contaminación, pueden ser utilizadas para construir diferentes índices bióticos, capaces de brindar información de la calidad del ambiente, como por ejemplo el Biological Monitoring Working Party (BMWP) y el Average Score Per Taxon (ASPT), que se basan en un listado de 85 familias de macroinvertebrados con un grado conocido de tolerancia y sensibilidad a la contaminación orgánica; o el EPT (Ephemeroptera, Plecoptera y Trichoptera) que se basa en la abundancia relativa de los tres órdenes de insectos más sensibles a la contaminación (Armitage et al. 1983, Gray y Delaney 2008).

La Familia Chironomidae (Orden Díptera) es uno de los grupos de insectos del macrobentos más ampliamente distribuidos y abundantes en los cuerpos de agua dulce (Craston 1995, Medina y Paggi 2004, Donato et al. 2009) y es de gran interés para el completo conocimiento de los ecosistemas acuáticos continentales, por su considerable abundancia y riqueza específica y por su gran plasticidad ecológica, que le permite habitar en una gran variedad de ambientes (Paggi 2009, Zanotto-Arpellino et al. 2015). Los insectos quironómidos pueden explotar áreas con amplios gradientes ambientales, siendo muchas veces los únicos representantes en ambientes fuertemente contaminados, y tienen la particularidad de formar parte de distintas comunidades, tales como bentos, pleuston y perifiton (Marchese y Paggi 
2004). Sus estados inmaduros (larvas y pupas) juegan un rol importante en la dinámica de la comunidad bentónica de la mayoría de los cuerpos de agua (Paggi 1999).

En las últimas décadas, los insectos quironómidos se han utilizado para caracterizar el estado ambiental de los sistemas fluviales en todo el mundo, particularmente cuando se identifican a nivel de género y especie (Cranston 1995, Epele et al. 2012). En la Argentina, se ha comenzado más recientemente a considerar a los macroinvertebrados bentónicos, y entre ellos a los insectos quironómidos, en los estudios de contaminación orgánica e inorgánica, tendientes a la confección de índices biológicos como herramientas para la medición de calidad del agua (Paggi 1999).

En la Patagonia, se registran hasta la actualidad 9 subfamilias de Chironomidae, representadas por 53 de los 111 géneros conocidos para América del Sur. Del total de géneros de la Patagonia, el 20\% es endémico (Donato et al., 2009). La gran diversidad exhibida por este grupo en la región, sumado a su capacidad de habitar en un amplio gradiente de condiciones ambientales, permite considerar su utilidad como bioindicador de la calidad del agua.

Los ríos urbanos figuran entre los sistemas más degradados porque son impactados por varias fuentes de contaminación tanto puntuales como difusas. Reciben los desagües pluviales, cloacales e industriales, y por lo tanto, una alta carga de contaminantes, que incluyen a los nutrientes y materia orgánica, cuyo procesamiento es crítico para la calidad del agua de estos ríos (Pave y Marchese 2005). Particularmente, la cuenca superior del Río Gallegos presenta un grado relativamente alto de antropización, generado por la explotación minera de carbón (principal actividad productiva de la zona), por el crecimiento urbano sin planificación y por la utilización de espacios generados para la cría extensiva de ganado (Asueta 2016).

Estudios previos realizados en la región, utilizando la comunidad de macroinvertebrados bentónicos y aplicando índices bióticos a nivel taxonómico de órdenes y familias, permitieron determinar la existencia de procesos de impacto antrópico y degradación en los ambientes acuáticos de la cuenca (Asueta et al. 2013, Asueta et al. 2014 a, Asueta et al. 2014 b). Estos estudios, pusieron también en evidencia la necesidad de trabajar a niveles taxonómicos más precisos, que involucran la identificación a nivel de género y especie de algunos de los organismos predominantes en la comunidad bentónica. En función de esto, la presente propuesta propone trabajar con los insectos de la Familia Chironomidae para ahondar en el conocimiento taxonómico del grupo y evaluar su potencial como indicador de la calidad ambiental en la región. La información obtenida, permitirá ajustar y mejorar la eficiencia de los índices bióticos de calidad ambiental diseñados y adaptados para su uso en la región.

\section{Objetivo general del trabajo:}

- Trabajar con los insectos de la Familia Chironomidae para ahondar en el conocimiento taxonómico del grupo y evaluar su potencial como indicador de la calidad ambiental en la región

\section{MATERIALES Y MÉTODOS}

El área de estudio se localiza en la Cuenca del Río Gallegos (Provincia de Santa Cruz), el curso de agua más importante del sur de la provincia (Figura 1 y 2). Esta cuenca se extiende de Oeste a Este unos $300 \mathrm{~km}$ y desagua en el Océano Atlántico, abarcando una superficie de $8400 \mathrm{~km}^{2}$.

Sobre la cuenca se localizan tres localidades de la provincia de Santa Cruz: Río Turbio, 28 de Noviembre y la ciudad de Río Gallegos, capital de la provincia. Esta es quizás una de las cuencas con mayor incidencia y superposición de actividades productivas y urbanas en la provincia, lo que hace particularmente relevante su estudio e interesante su análisis por la complejidad que presenta.

Los muestreos de los macroinvertebrados bentónicos se realizaron en 7 sitios de estudio (Fig. 1): 5 de ellos correspondientes a zonas con diferente grado de perturbación de origen 
antrópico (sitios TP: Planta de tratamiento de Efluentes Cloacales Río Turbio, MA: frente a Mina de Carbón, DM: Después de Usina termoeléctrica, GC: Estancia Glencross y MCH: Sitio Estancia Morro Chico y 2 considerados sitios de referencia (Sitio AP: Arroyo primavera y Sitio SR: Rio Rubens), por no presentar impactos sostenidos de actividad humana. Los muestreos biológicos y la medición de las variables ambientales se efectuaron simultáneamente y en forma estacional durante 2017-2018. Si bien durante el 2018 se concluyó con las cuatros campañas de toma de muestras, en este estudio, se trabajaron los datos colectados durante la estación de otoño 2017.

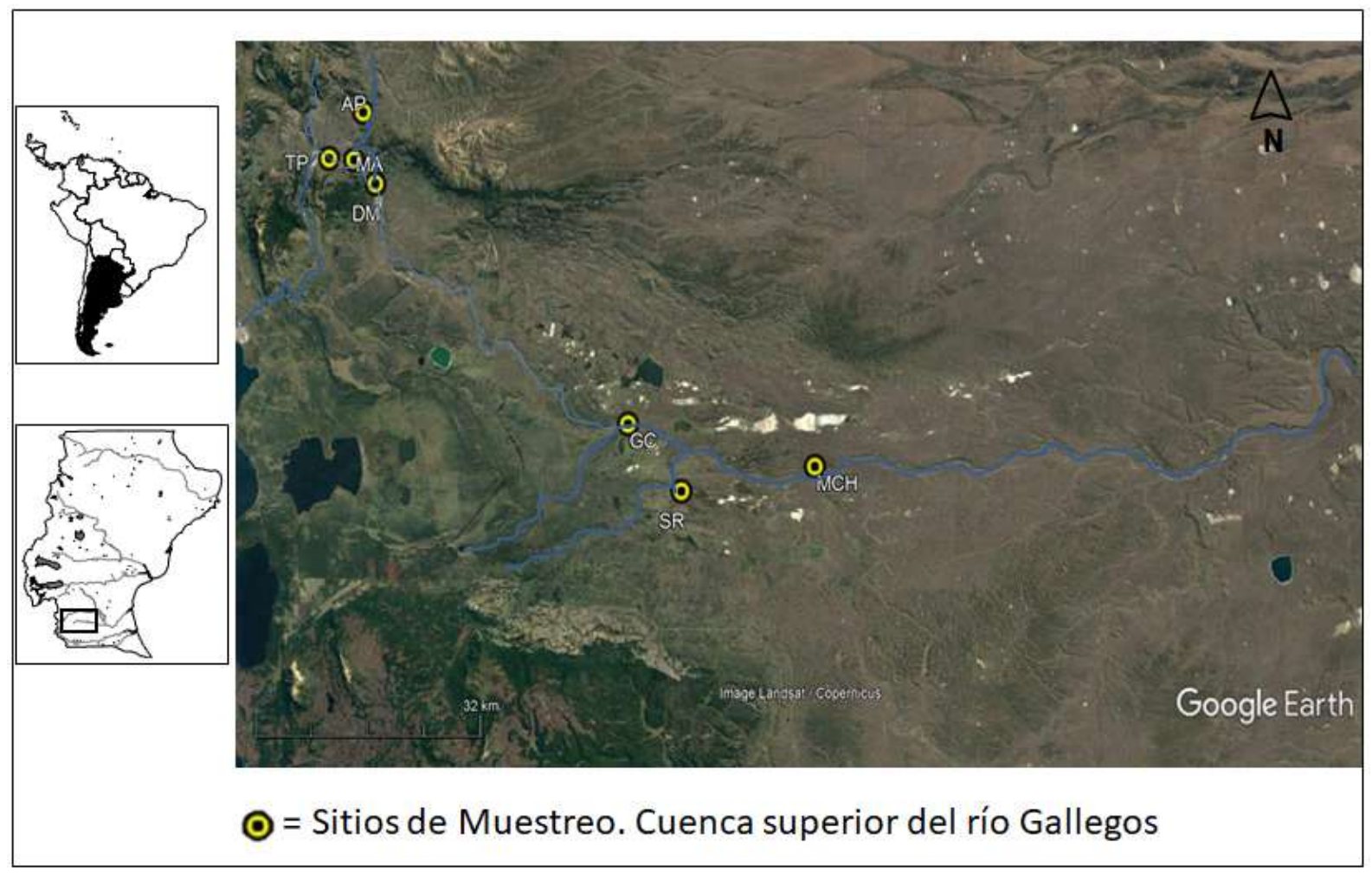

Figura 1. Área de estudio y puntos de muestreo.

\section{MUESTREO Y ANÁLISIS}

Se utilizó una sonda multiparamétrica (Horiba) para la medición in situ de los parámetros ambientales del agua tales como: $\mathrm{pH}$, sólidos disueltos totales, turbidez, temperatura, oxígeno disuelto y conductividad.

Las muestras de macroinvertebrados, fueron tomadas paralelas a la margen del río a escasa profundidad $(<30 \mathrm{~cm})$ en cada uno de los sitios, utilizando una red tipo Surber con malla de $500 \mu \mathrm{m}$ y un marco metálico para delimitar un área de muestreo de $0,25 \mathrm{~m}^{2}$ sobre el fondo. El sustrato fue removido con pala y en forma manual durante un minuto.

El material recolectado fue conservado en el campo con alcohol al 70\%, posteriormente lavado y separado del residuo en laboratorio. Utilizando un tamiz con malla de 500 micrones de abertura. Los organismos fueron contabilizados e identificados hasta nivel taxonómico más bajo posible, utilizando claves actualizadas (Domínguez y Fernández, 2009). Para la determinación de los quironómidos en particular, se efectuaron preparados permanentes y transitorios utilizando la metodología descripta por Paggi (2001), para su observación en microscopio binocular e identificación a nivel de género utilizando claves taxonómicas actualizadas. 


\section{PREPARACIONES MICROSCÓPICAS}

En laboratorio se separaron las larvas de Chironomidae del resto de la muestra y se conservaron en alcohol etílico al $70 \%$ para identificación y recuento. El material fue identificado hasta el máximo nivel taxonómico de determinación posible, utilizando la clave de Paggi (2009).

Se realizaron preparaciones tanto permanentes como transitorias con las larvas de todos los organismos de la familia Chironomidae.

Según Paggi (2009), el proceso consiste en el aclarado de las larvas con una solución de OHK al $10 \%$, lavado en solución de agua destilada y gotas de ácido acético glacial por algunos minutos y montaje de piezas en preparaciones transitorias o definitivas. Se utilizaron ambos tipos de preparaciones para poner en práctica las técnicas aprendidas durante la pasantía.

El montaje transitorio permitió montar varias muestras en poco tiempo, utilizando líquido de Faure (solución transparente utilizada para fijar y montar). El montaje definitivo, consistió en la deshidratación de las larvas pasándolas a través de una serie de alcoholes $\left(80^{\circ}, 96^{\circ} \mathrm{y}\right.$ $100^{\circ}$ de etanol), para luego fijar las piezas con bálsamo de Canadá.

Ambos preparados concluyeron con un secado en estufa a $45^{\circ} \mathrm{C}$ hasta que la solución se endureció.

La larva se monta con vista dorsal y se trata de trabajar con individuos en estadio larval 4 (de mayor tamaño) para poder observar todas las estructuras.

\section{ANÁLISIS ESTADÍSTICOS}

Para cada muestra de macroinvertebrados se calculó utilizando el paquete estadístico InfoStat 2017, la abundancia total y relativa (\%) de cada taxón; la riqueza de taxones (S), definida como el número de taxones presentes en la muestra, la dominancia (D), la diversidad (H') a partir del índice de diversidad de Shannon-Wiener (utilizando logaritmo en base 2) y la equitatividad con el índice de Pielou.

Los datos de abundancia de los diferentes taxones se analizaron mediante estadística multivariada, utilizando técnicas de agrupamiento (Hierarchical agglomerative clustering, HAC) y de ordenamiento no paramétrico (Multidimensional Scaling, MDS), por medio del software PRIMER versión 6.1. La significancia estadística de los agrupamientos entre muestras se corroboro mediante el test ANOSIM, para poner a prueba la hipótesis de diferencias en la composición de la comunidad entre sitios de muestreo (Clarke y Warwick, 2001).

La dominancia (D) se calculó como:

Dónde:

$$
\mathrm{D}=(\mathrm{n} / \mathrm{N}) \times 100
$$

- $\mathrm{n}=$ número total de individuos del taxón considerado.

- $\quad \mathrm{N}=$ número total de individuos de todos los taxones.

El índice de Shannon-Wiener se calculó a través de la siguiente fórmula:

Dónde:

$$
\mathrm{H}^{\prime}=-\sum \text { pi ln pi }
$$

- $\quad \mathrm{pi}=\mathrm{ni} / \mathrm{N}$; número de organismos de la especie i /número total de organismos.

- $\quad n i=$ número de organismos de la especie $\mathrm{i}$.

- $\quad \mathrm{N}=$ número total de organismos.

La riqueza específica (S) fue calculada como el número total de taxa presentes en las muestras. 
La equitatividad de Pielou $\left(\mathrm{J}^{\prime}\right)$, que representa la uniformidad en la distribución numérica entre las diferentes especies del conjunto estudiado en cada período de muestreo, se calculó con la siguiente fórmula:

Dónde:

$$
\mathrm{J}^{\prime}=\mathrm{H}^{\prime} / \ln \mathrm{S}
$$

- $\quad$ S es el número máximo de especies en la muestra.

- $\quad H^{\prime}$ es el índice de Shannon -Wiener.

\section{PARÁMETROS AMBIENTALES}

\section{RESULTADOS}

Tabla 1. Parámetros ambientales tomados In Situ en los distintos sitios de muestreo. Estación otoño.

\begin{tabular}{|c|c|c|c|c|c|c|c|}
\hline Sitio & Coordenadas & $\begin{array}{c}\text { Temperatura } \\
\left({ }^{\circ} \mathrm{C}\right)\end{array}$ & $\mathrm{pH}$ & Oxígeno Disuelto (mg/l) & $\begin{array}{c}\text { Sólidos } \\
\text { disueltos } \\
\text { totales }(\mathrm{g} / \mathrm{l})\end{array}$ & $\begin{array}{c}\text { Conductividad } \\
\text { eléctrica }(\mathrm{ms} / \mathrm{cm})\end{array}$ & $\begin{array}{c}\text { Turbidez } \\
\text { (NTU) }\end{array}$ \\
\hline AP & $51^{\circ} 29^{\prime} 02.9^{\prime \prime S} 72^{\circ} 14^{\prime} 35.4^{\prime \prime O}$ & 1,96 & 7,5 & 11,9 & 0,08 & 0,12 & 1,90 \\
\hline TP & $51^{\circ} 32^{\prime} 07.0^{\prime \prime} \mathrm{S} 72^{\circ} 18^{\prime} 30.3^{\prime \prime O}$ & 5,29 & 7,75 & 9,4 & 0,40 & 0,63 & 141,00 \\
\hline $\mathrm{MA}$ & $51^{\circ} 32^{\prime} 12.2^{\prime \prime S} 72^{\circ} 15^{\prime} 37.5^{\prime \prime O}$ & 2,46 & 7,23 & 9,8 & 0,13 & 0,20 & 7,30 \\
\hline $\mathrm{DM}$ & $51^{\circ} 33^{\prime} 46.8^{\prime \prime} \mathrm{S} 72^{\circ} 13^{\prime} 00.8^{\prime \prime} \mathrm{O}$ & 2,71 & 7,47 & 9,8 & 0,16 & 0,25 & 24,90 \\
\hline $\mathrm{GC}$ & $51^{\circ} 49^{\prime} 25^{\prime \prime}$ S $71^{\circ} 42^{\prime} 14.38^{\prime \prime} 0$ & 2,4 & 7,75 & 8,5 & 0,19 & 0,29 & 20,10 \\
\hline SR & $51^{\circ} 53^{\prime} 48.2^{\prime \prime} \mathrm{S} 71^{\circ} 35^{\prime} 39.4^{\prime \prime} \mathrm{O}$ & 1,98 & 6,93 & 10,5 & 0,04 & 0,05 & 2,20 \\
\hline $\mathrm{MCH}$ & $51^{\circ} 51^{\prime} 57.7^{\prime \prime S} 71^{\circ} 19^{\prime} 34.1^{\prime \prime O}$ & 1,5 & 7,17 & 10,4 & 0,06 & 0,09 & 4,80 \\
\hline
\end{tabular}

En la Tabla 1, podemos observar las mediciones de parámetros ambientales tomados en los sitios de muestreo, durante la campaña de otoño.

La temperatura del agua presento valores que oscilaron entre 1,5 y $7,7^{\circ} \mathrm{C}$. El oxígeno disuelto varío desde $8,5 \mathrm{mg} / \mathrm{l}$ en el sitio GC a $11,9 \mathrm{mg} / \mathrm{l}$ en el sitio AP. A su vez, TP y SR, presentaron el valor más bajo y más alto de solidos disueltos totales, con $0,40 \mathrm{~g} / \mathrm{l}$ y $0,08 \mathrm{~g} / \mathrm{l}$ respectivamente.

Los valores de $\mathrm{pH}$ son neutros, oscilando entre 6.93 y 7.75 .

Los valores máximos de turbidez registrados se encontraron en el sitio TP con un valor de 141 NTU.

La conductividad eléctrica presento un rango de valores que oscilaron entre $0,05 \mathrm{~ms} / \mathrm{cm}$ y $0,63 \mathrm{~ms} / \mathrm{cm}$.

\section{MACROINVERTEBRADOS BENTÓNICOS}

La fauna de macroinvertebrados bentónicos otoño estuvo representada por organismos de 30 taxa, con una densidad media de 5341 individuos por $\mathrm{m}^{2}$.

Los grupos de mayor dominancia estuvieron constituidos por la familia Leptophlebiidae (Ephemeroptera) con un 19,76\%, seguido por Hyalellidae (Amphipoda) con un $17,70.08 \%$ y Clase Oligochaeta con un 16,89 \%. En cuanto a los Insectos pertenecientes al orden Díptera, podemos destacar la dominancia de la Familia Chironomidae representada en un 12,42\%.

La mayor riqueza de macroinvertebrados se registró en Sitio SR con 19 taxones identificados, el menor en el Sitio TP donde se contabilizaron tres taxa (Tabla 2). Según la Figura 1, en relación a la riqueza específica, los sitios AP (Arroyo Primavera) y SR (Río Rubens) son significativamente distintos del Sitio MA (Punto frente a la Mina de Carbón). 
Tabla 2. Medidas de resumen para la Riqueza específica, Abundancia, $S, \mathbf{H}^{\prime}$ y J'.

\begin{tabular}{|ccccc|}
\hline Sitios & Riqueza $(\mathrm{S})$ & Abundancia & Equitatividad $\left(\mathrm{J}^{\prime}\right)$ & Diversidad $\left(\mathrm{H}^{\prime}\right)$ \\
\hline AP & 11,5 & 1360 & 0,67 & 1,62 \\
\hline TP & 3,5 & 90 & 0,56 & 0,71 \\
\hline MA & 1,75 & 689 & 0,03 & 0,02 \\
\hline DM & 2,75 & 885 & 0,34 & 0,27 \\
\hline GC & 7 & 584 & 0,51 & 1,01 \\
\hline SR & 16,25 & 4227 & 0,67 & 1,86 \\
\hline MCH & 8,75 & 1513 & 0,75 & 1,62 \\
\hline
\end{tabular}

La abundancia relativa promedio más baja se registró en el Sitio TP $\left(90 \mathrm{ind} / \mathrm{m}^{2}\right)$ y la más alta en el sitio SR (4227 ind $/ \mathrm{m}^{2}$ ). Se registraron diferencias significativas entre los sitios SR y TP (Fig. 2).

Los anélidos oligoquetos se encuentran presentes en todas las estaciones, y en algunos sitios con baja diversidad, como el MA y DM representan un 99 y $97 \%$ de dominancia respectivamente (Tabla 3 ).
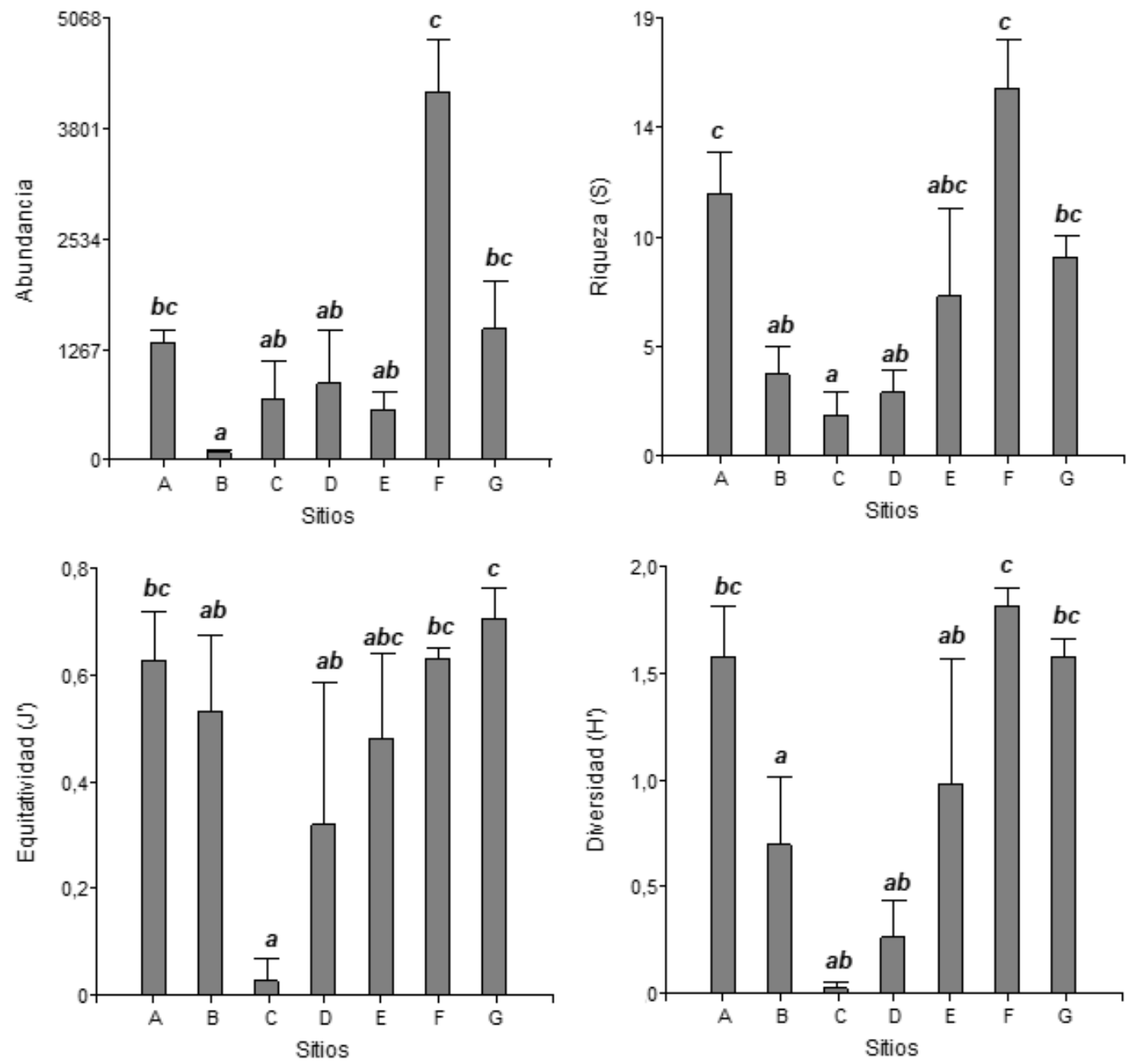

Figura 2. Índices comunitarios calculados para cada sitio. Valores promedio ( \pm desviación típica). Medias con una letra común no son significativamente diferentes (Test de Kruskall-Wallis p>0,05).

La diversidad mostro diferencias significativas entre el sitio TP y el SR. La diversidad mínima fue de 0,02 bits/ind en el sitio MA (Frente a mina), seguido por el sitio DM (después de mina) con 0,07 bits/ind. La máxima diversidad se obtuvo en los sitios SR (Río Rubens), MCH (Morro Chico) y AP (Arroyo Primavera) 1,95; 1,67 y 1,92 puntos respectivamente. 
La equitatividad registró su valor mínimo en el Sitio TP $(0,02)$ y su valor máximo en el sitio MCH (Morro Chico) con 0,80 puntos. El índice de equitatividad exhibe diferencias significativas entre los puntos MA y MCH (Fig. 2).

Tabla 3. Dominancia (\%) por sitio de los macroinvertebrados bentónicos presentes en los sitios. Estación otoño.

\begin{tabular}{|c|c|c|c|c|c|c|c|}
\hline 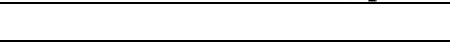 & \multicolumn{7}{|c|}{ SITIOS } \\
\hline TAXÓN & $\mathrm{AP}$ & TP & MA & $\mathrm{DM}$ & GC & SR & $\mathrm{MCH}$ \\
\hline Lymnaeidae (mollusca gastropoda) & 0,44 & 0,00 & 0,00 & 0,00 & 18,15 & 9,77 & 29,87 \\
\hline Chilinidae (mollusca gastropoda) & 0,00 & 0,00 & 0,00 & 0,00 & 2,05 & 0,31 & 9,25 \\
\hline Hydrobiidae (mollusca gastropoda) & 0,00 & 0,00 & 0,00 & 0,00 & 0,00 & 0,47 & 0,00 \\
\hline Sphaeriidae (mollusca bivalvia) & 0,00 & 0,00 & 0,00 & 0,00 & 0,00 & 0,12 & 0,00 \\
\hline Gripopterygidae (Plecoptera) & 5,29 & 0,00 & 0,00 & 0,00 & 3,94 & 0,62 & 6,74 \\
\hline Leptophlebiidae (Ephemeroptera) & 36,18 & 0,00 & 0,00 & 0,00 & 8,73 & 21,65 & 19,43 \\
\hline Baetidae (Ephemeroptera) & 0,00 & 0,00 & 0,00 & 0,00 & 0,17 & 0,02 & 0,00 \\
\hline Hydroptilidae (Trichoptera) & 11,69 & 0,00 & 0,29 & 1,58 & 0,17 & 1,42 & 0,00 \\
\hline Hidrobyosidae (Trichoptera) & 0,00 & 0,00 & 0,00 & 0,00 & 0,00 & 0,38 & 0,00 \\
\hline Limnephilidae (Trichoptera) & 0,00 & 0,00 & 0,00 & 0,00 & 0,00 & 0,09 & 0,00 \\
\hline Philophotamidae (Trichoptera) & 0,22 & 0,00 & 0,00 & 0,00 & 0,00 & 0,07 & 0,00 \\
\hline Glossosomatidae (Trichoptera) & 0,00 & 0,00 & 0,00 & 0,00 & 0,34 & 0,12 & 0,00 \\
\hline Oligochaeta (Annelida) & 6,69 & 61,11 & 99,42 & 97,40 & 8,90 & 5,30 & 0,66 \\
\hline Glosshiphonidae (Annelida) & 0,15 & 0,00 & 0,00 & 0,00 & 0,34 & 1,85 & 1,85 \\
\hline Hydrachnidae (Acari) & 0,29 & 0,00 & 0,00 & 0,00 & 0,17 & 0,02 & 0,00 \\
\hline Belastomatidae (Hemiptera) & 0,07 & 0,00 & 0,00 & 0,00 & 0,00 & 0,00 & 0,00 \\
\hline Macroveelidae (Hemiptera) & 0,00 & 0,00 & 0,15 & 0,00 & 0,00 & 0,00 & 0,00 \\
\hline Corixidae (Hemiptera) & 0,00 & 0,00 & 0,00 & 0,00 & 0,00 & 0,00 & 0,07 \\
\hline Collembola & 0,00 & 2,22 & 0,15 & 0,11 & 0,51 & 0,00 & 0,00 \\
\hline Chironomidae (Diptera) & 17,65 & 2,22 & 0,00 & 0,90 & 25,17 & 16,63 & 0,07 \\
\hline Empididae (Diptera) & 1,69 & 0,00 & 0,00 & 0,00 & 0,00 & 0,07 & 0,00 \\
\hline Dolichopididae (Diptera) & 0,00 & 0,00 & 0,00 & 0,00 & 0,00 & 0,05 & 0,00 \\
\hline Tipulidae (Diptera) & 0,66 & 0,00 & 0,00 & 0,00 & 1,71 & 0,07 & 0,00 \\
\hline Ephydridae (Diptera) & 0,07 & 0,00 & 0,00 & 0,00 & 0,00 & 0,00 & 0,00 \\
\hline Psychodidae (Diptera) & 0,00 & 32,22 & 0,00 & 0,00 & 0,00 & 0,00 & 0,00 \\
\hline Tabanidae (Diptera) & 0,00 & 1,11 & 0,00 & 0,00 & 0,00 & 0,05 & 0,00 \\
\hline Ceratopogonidae (Diptera) & 0,00 & 1,11 & 0,00 & 0,00 & 0,00 & 0,00 & 0,00 \\
\hline Simulidae (Diptera) & 0,00 & 0,00 & 0,00 & 0,00 & 0,00 & 0,31 & 0,13 \\
\hline Hyalellidae (Amphipoda) & 17,06 & 0,00 & 0,00 & 0,00 & 28,77 & 17,01 & 30,27 \\
\hline Elmidae (Coleoptera) & 1,84 & 0,00 & 0,00 & 0,00 & 0,86 & 23,61 & 1,65 \\
\hline
\end{tabular}

A partir de los análisis de ordenamiento y agrupamiento (Fig. 3 y 4) aplicados al muestreo de otoño, se pudieron identificar tres grupos de muestras. Este agrupamiento fue estadísticamente significativo según el test de ANOSIM ( $\mathrm{R}$ global $=0,799$, Nivel de significancia del estadístico muestral: $0,1 \%$ ).

El análisis SIMPER permitió diferenciar que taxa aportan o contribuyen a la similitud de cada uno de los grupos. Se trabajaron los datos de abundancia con una similitud de $40 \%$. 
Tabla 4. Análisis SIMPER. Principales taxones que contribuyen en la similitud de los grupos. $\mathrm{AP}=$ abundancia promedio (datos transformados por raíz cuarta); $\mathrm{SP}=$ similitud promedio; $\mathrm{DE}=$ desviación estándar; $\mathrm{CP}=$ contribución (\%); $\mathrm{PA}=$ porcentaje acumulado

\begin{tabular}{|c|c|c|c|c|c|c|}
\hline Grupos & Taxa & $A P$ & $S P$ & $D E$ & $C P$ & $P A$ \\
\hline \multirow{8}{*}{$\begin{array}{c}\text { Grupo A } \\
\text { Similitud: } \\
79,93 \%\end{array}$} & Hyalellidae (Amphipoda) & 3,88 & 11,89 & 23,51 & 14,88 & 14,88 \\
\hline & Leptophlebiidae (Ephemeroptera) & 4,39 & 11,54 & 4,21 & 14,44 & 29,32 \\
\hline & Hydroptilidae (Trichoptera) & 3,53 & 10,88 & 11,13 & 13,62 & 42,94 \\
\hline & Chironomidae (Diptera) & 3,6 & 9,52 & 4,79 & 11,91 & 54,85 \\
\hline & Oligochaeta (Annelida) & 3,05 & 9,14 & 29,97 & 11,44 & 66,29 \\
\hline & Gripopterygidae (Plecoptera) & 2,83 & 8,41 & 4,18 & 10,52 & 76,81 \\
\hline & Elmidae (Coleoptera) & 2,12 & 5,8 & 5,27 & 7,26 & 84,07 \\
\hline & Empididae & 2,06 & 5,51 & 5,78 & 6,9 & 90,97 \\
\hline \multirow{2}{*}{$\begin{array}{c}\text { Grupo B } \\
\text { Similitud: } \\
69,10 \%\end{array}$} & Oligochaeta (Annelida) & 2,72 & 39,67 & 4,78 & 57,41 & 57,41 \\
\hline & Psychodidae (Diptera) & 2,06 & 23,79 & 7,5 & 34,43 & 91,84 \\
\hline $\begin{array}{c}\text { Grupo C } \\
\text { Similitud: } \\
61,75 \% \\
\end{array}$ & Oligochaeta (Annelida) & 4,34 & 61,75 & 3,14 & 100 & 100 \\
\hline \multirow{3}{*}{$\begin{array}{c}\text { Grupo D } \\
\text { Similitud: } \\
62,14 \%\end{array}$} & Oligochaeta (Annelida) & 4,55 & 42 & 5,07 & 67,59 & 67,59 \\
\hline & Hydroptilidae (Trichoptera) & 1,53 & 11,08 & 0,9 & 17,82 & 85,42 \\
\hline & Chironomidae (Diptera) & 1,32 & 9,06 & 0,85 & 14,58 & 100 \\
\hline \multirow{4}{*}{$\begin{array}{c}\text { Grupo E } \\
\text { Similitud: } \\
44,63 \%\end{array}$} & Hyalellidae (Amphipoda) & 3,26 & 15,96 & 3,35 & 35,77 & 35,77 \\
\hline & Chironomidae (Diptera) & 2,99 & 13,68 & 2,1 & 30,65 & 66,42 \\
\hline & Oligochaeta (Annelida) & 2,01 & 5,7 & 0,89 & 12,77 & 79,19 \\
\hline & Gripopterygidae (Plecoptera) & 1,72 & 5,54 & 0,87 & 12,41 & 91,6 \\
\hline \multirow{11}{*}{$\begin{array}{c}\text { Grupo F } \\
\text { Similitud: } \\
80,06\end{array}$} & Elmidae (Coleoptera) & 5,54 & 10,38 & 10,63 & 12,97 & 12,97 \\
\hline & Hyalellidae (Amphipoda) & 5,13 & 9,78 & 14,48 & 12,22 & 25,19 \\
\hline & Leptophlebiidae (Ephemeroptera) & 5,35 & 9,66 & 8,02 & 12,07 & 37,26 \\
\hline & Chironomidae (Diptera) & 5,02 & 9,08 & 9,26 & 11,34 & 48,6 \\
\hline & Lymnaeidae (mollusca gastropoda & 4,38 & 8,02 & 4,5 & 10,01 & 58,61 \\
\hline & Oligochaeta (Annelida) & 3,8 & 6,99 & 10,91 & 8,74 & 67,35 \\
\hline & Glosshiphonidae (Annelida) & 2,95 & 5,66 & 11,11 & 7,06 & 74,41 \\
\hline & Hydroptilidae (Trichoptera) & 2,62 & 4,47 & 9,68 & 5,58 & 79,99 \\
\hline & Hidrobyosidae (Trichoptera) & 1,98 & 3,81 & 12,53 & 4,76 & 84,75 \\
\hline & Gripopterygidae (Plecoptera) & 2,12 & 3,68 & 6,63 & 4,6 & 89,35 \\
\hline & Chilinidae (mollusca gastropoda & 1,74 & 2,94 & 17,66 & 3,68 & 93,03 \\
\hline \multirow{7}{*}{$\begin{array}{c}\text { Grupo G } \\
\text { Similitud: } \\
83,94 \%\end{array}$} & Hyalellidae (Amphipoda) & 4,42 & 14,77 & 10,18 & 17,59 & 17,59 \\
\hline & Lymnaeidae (mollusca gastropoda & 4,41 & 14,67 & 8,94 & 17,48 & 35,07 \\
\hline & Leptophlebiidae (Ephemeroptera) & 3,96 & 13,47 & 10,07 & 16,05 & 51,12 \\
\hline & Gripopterygidae (Plecoptera) & 3,08 & 10,78 & 9,35 & 12,84 & 63,95 \\
\hline & Chilinidae (mollusca gastropoda & 3,23 & 10,46 & 7,9 & 12,46 & 76,42 \\
\hline & Glosshiphonidae (Annelida) & 2,22 & 7,43 & 8,52 & 8,85 & 85,27 \\
\hline & Elmidae (Coleoptera) & 2,04 & 6,36 & 6,91 & 7,58 & 92,85 \\
\hline
\end{tabular}




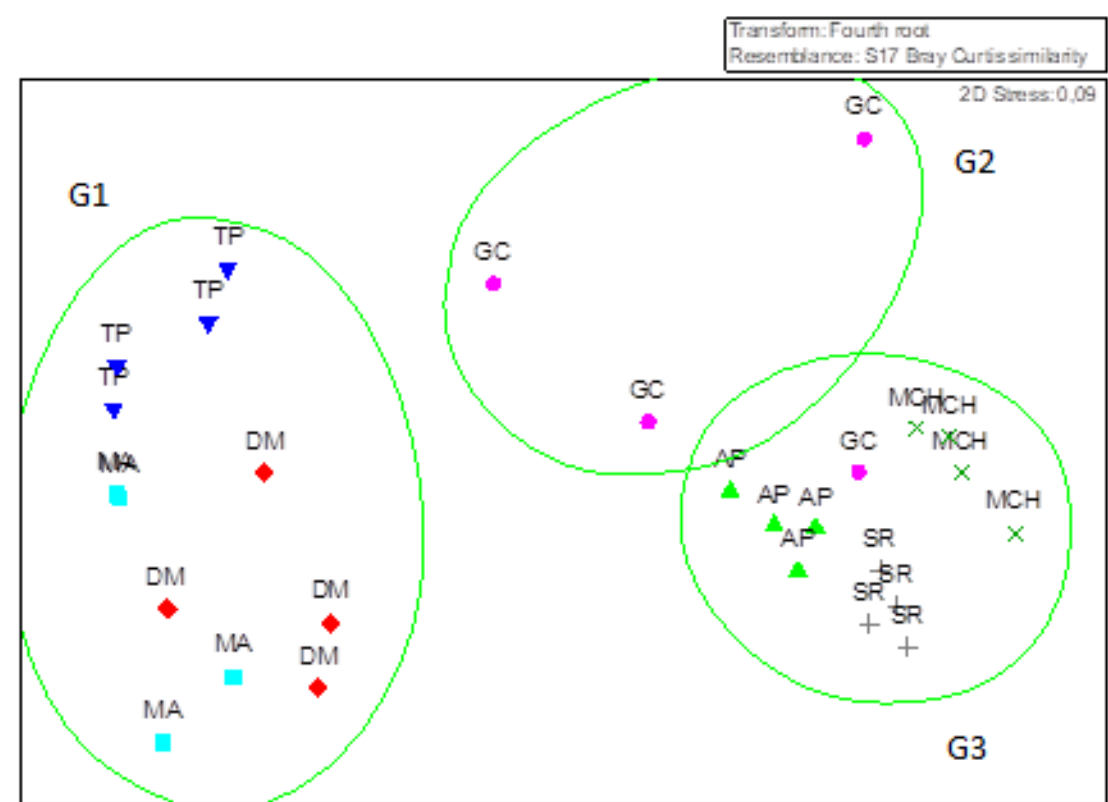

Figura 3. Análisis de Ordenamiento (MDS) para Muestreo de Estación Otoño.

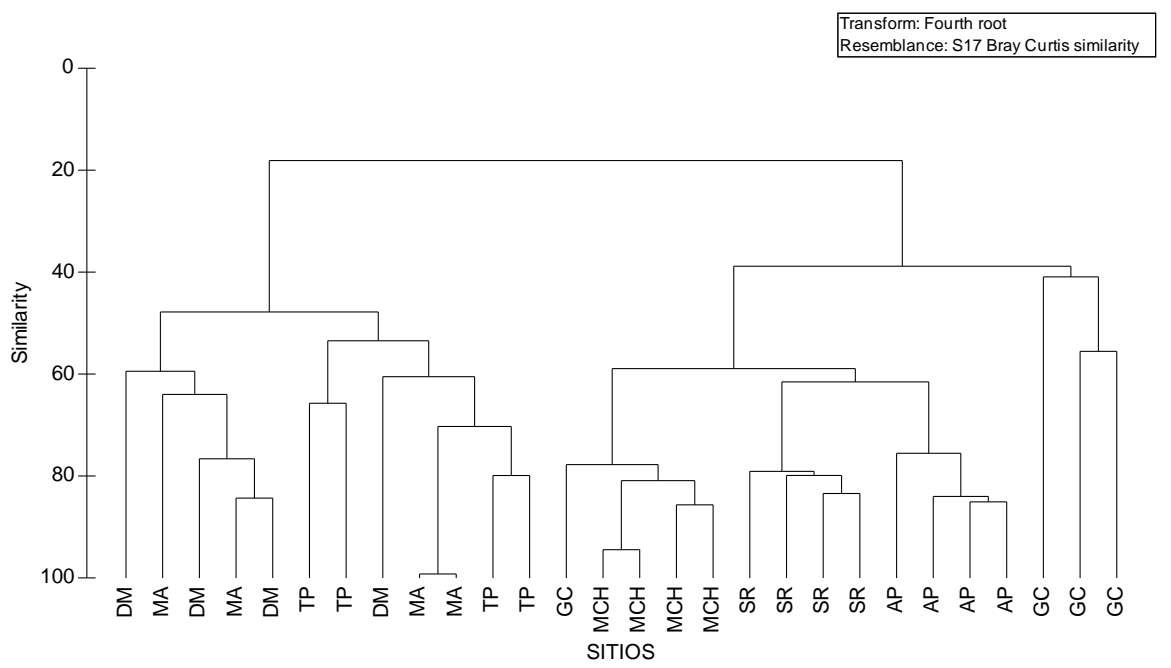

Figura 4. Análisis de Agrupamiento (HAC). Muestreo de macroinvertebrados bentónicos, Estación Otoño.

El Grupo 1 está integrado por las muestras de los sitios TP (planta de tratamiento), MA (frente a mina de carbón) y DM (después de Usina) caracterizadas por la presencia de anélidos oligoquetos con porcentajes altos de contribución a la similitud de las muestras mayores al $50 \%$.

El Grupo 2 se conforma por tres de las muestras del sitio GC (Glencross), representado por la presencia de oligoquetos, Insectos de la familia. Chironomidae y plecópteros de la familia Gripopterygidae.

Por último, el Grupo 3 integra a las muestras de los sitios AP (Arroyo Primavera), SR (Río Rubens) y MCH (Morro Chico), y una muestra del sitio GC. Este grupo se caracteriza por la presencia de anfípodos de la familia Hyalellidae, gasterópodos de la familia Lymnaeidae, coleópteros de la familia Elmidae, plecópteros de la familia Gripopterygidae, anélidos oligoquetos e insectos quironómidos fueron comunes a todas las muestras. En el caso de la muestra del sitio GC que se incluye en este grupo, el taxa que aporta el 35\% de similitud son los anfípodos de la familia Hyalellidae, seguido por los insectos de la familia Chironomidae con un $30 \%$. 
En relación a los insectos quironómidos, se identificaron un total de 10 géneros, de los cuales 5 pertenecen a la subfamilia Orthocladiinae, 2 de Tanypodinae y 3 de Chironominae. (Tabla $5)$.

\begin{tabular}{|c|c|c|}
\hline \multicolumn{3}{|c|}{ Argentina), para la estación de Otoño (Mayo de 2017). } \\
\hline Familia & Subfamilia & Género \\
\hline Chironomidae & Orthocladiinae & Orthocladius en parte/Cricotopus en parte \\
\hline \multicolumn{3}{|r|}{ Orthocladius en parte/Cricotopus en parte } \\
\hline & & Paratrichocladius \\
\hline \multicolumn{3}{|r|}{ Parametriocnemus } \\
\hline & & Allocladius \\
\hline \multicolumn{2}{|r|}{ Tanypodinae } & Pentaneura \\
\hline & & Apsectrotanypus \\
\hline \multicolumn{2}{|r|}{ Chironominae } & Tanytarsus \\
\hline \multicolumn{3}{|r|}{ Polypedilum } \\
\hline \multicolumn{3}{|r|}{ Apedilum } \\
\hline
\end{tabular}
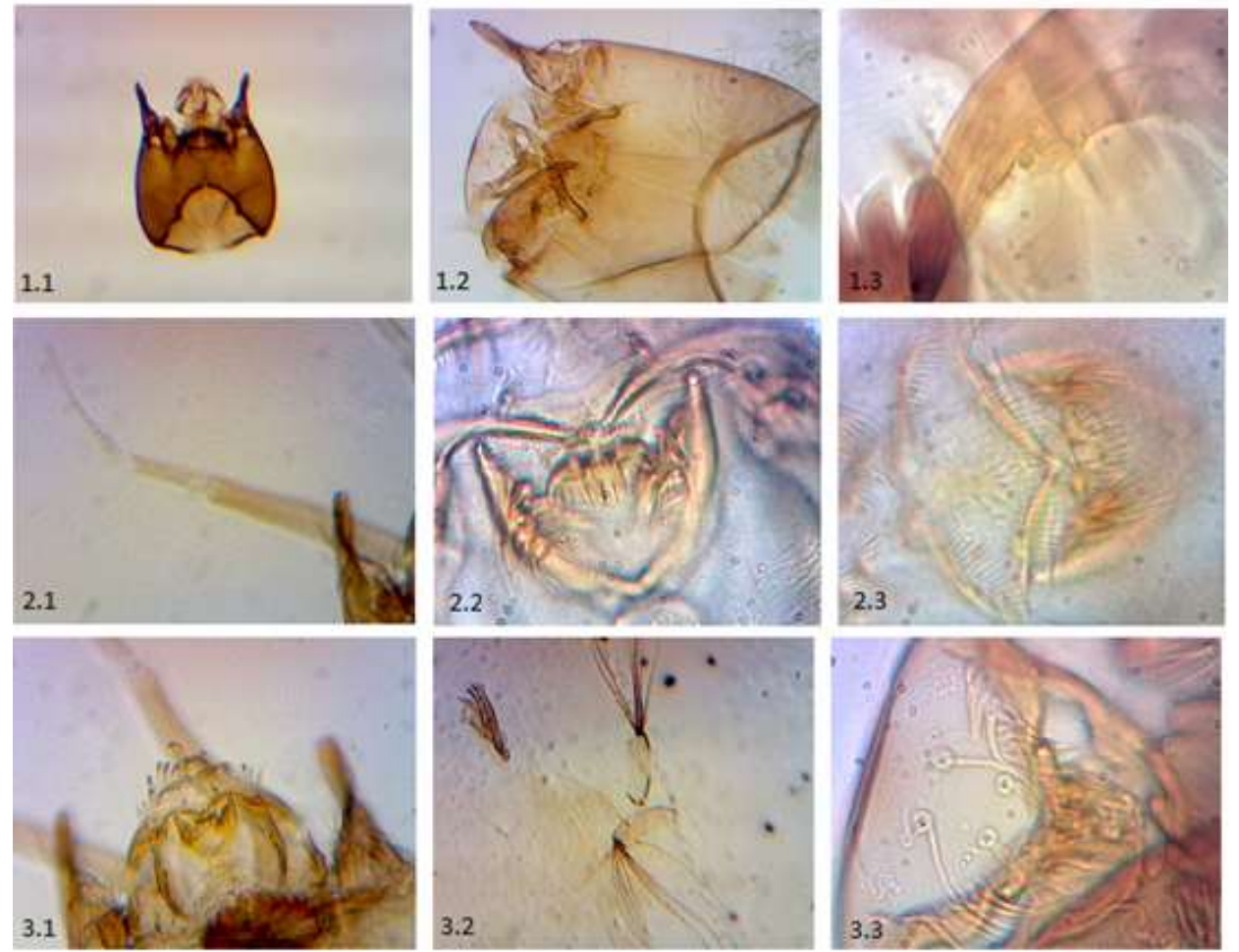

Figura 5. 1.1- Mentón de G. Alloclaudius. 1.2- Mentón G. Cricotopus.1.3-Mandíbula G. Pentaneura. 2.1- Pedúnculos y órganos de Lauterborn. 2.2. Peine de la epifaringe. 2.3. Peine de la epifaringe (II). 3.1- Peine de la Epifaringe. 3.2Procercos. 3.3- Seta I bífida.

\section{CONCLUSIONES}

En relación a los objetivos planteados para el desarrollo de la beca de posgrado, podemos afirmar que se concluyó con el acondicionamiento del material de las todas campañas de muestreo y con la caracterización de la comunidad de macroinvertebrados bentónicos para la aplicación de los índices comunitarios empleados para la comparación entre sitios. Cabe destacar, que a los efectos del período de acción de la beca (10 meses), solo se trabajó con los datos de la campaña de otoño.

- La comunidad de macroinvertebrados para los 7 sitios, muestreados durante el otoño, 
está compuesta por 30 taxa.

- En este trabajo se aporta información taxonómica a nivel de género de la familia Chironomidae, la cual no había sido descripta hasta el presente para la zona de estudio.

- Se espera que a través de la caracterización de todas las estaciones restantes e identificando los insectos quironómidos a nivel de género, se determinen los ensambles de Chironomidae bentónicos y su variación entre los distintos sitios.

Conclusiones por sitio:

Sitios de referencia o blanco

SR (Río Rubens): Los valores más altos de diversidad se registraron en los puntos más alejados de la actividad antrópicas, considerados como de referencia. Se hallaron bien representados los órdenes de plecópteros, efemerópteros y tricópteros, considerados indicadores de buena calidad de agua. Los moluscos gasterópodos y moluscos bivalvos, se registraron en mayores proporciones en este sitio. Los coleópteros (familia Elmidae) mostraron una dominancia del $23 \%$, con respecto al resto de los sitios. Estos organismos también se consideran dentro de los rankings como indicadores de aguas limpias junto con las familias de EPT. En relación a los insectos quironómidos, se agrupan junto con los oligoquetos, como organismos tolerantes a determinados disturbios antrópicos. Sin embargo, la mayor abundancia relativa de estos insectos, se presentó con un $63 \%$ en el sitio SR tomado como punto de referencia.

AP (Arroyo Primavera): Los valores más altos de diversidad ( $\left.\mathrm{H}^{\prime}\right)$ se registraron en los puntos más alejados de las actividades antrópicas.

Zonas con diferente grado de perturbación de origen antrópico

MCH (Morro Chico): Los valores más altos de diversidad se registraron en los puntos más alejados de las actividades antrópicas. Los moluscos gasterópodos y moluscos bivalvos, se registraron en mayores proporciones en este sitio. Los organismos de la familia Chilinidae estuvieron presentes con un $84 \%$, con respecto a los otros sitios.

GC (Glencross): Presenta el valor más bajo de Oxígeno disuelto, sin embargo, se mantiene dentro de los valores aptos para el desarrollo de los organismos acuáticos.

MA (frente a la mina de carbón): Sitio cercano a zonas con disturbios antrópicos. Presentan los valores más bajos de equitatividad, diversidad y riqueza, con dominancias altas (> 97\%) de anélidos oligoquetos. Se registraron los valores más bajos de diversidad (H') asociados a zonas con diferente grado de alteración.

DM (Sitio ubicado poco después de la usina termoeléctrica): sitio cercano a zonas con disturbios antrópicos. Presenta los valores más bajos de equitatividad, diversidad y riqueza, con dominancias altas (> 97\%) de anélidos oligoquetos. Se registraron los valores más bajos de diversidad (H') asociado a zonas con diferente grado de alteración.

TP (Planta de tratamiento de residuos cloacales): Sitio antropizado. Presenta los valores más altos de sólidos disueltos totales, turbidez y conductividad eléctrica. Se registraron los valores más bajos de diversidad (H') asociados a zonas con diferente grado de alteración. 


\section{BIBLIOGRAFÍA}

ALBA-TECEDOR, J. (1996). IV Simposio del agua en Andalucía (SIAGA). Macroinvertebrados acuáticos y calidad de las auas de los ríos (págs. 203-213). Andalucía: Almería.

ASUETA, R., SÚNICO, A., VALLEJOS, J., MARTIN, J., GUGLIELMINETTI, G., y AGUAS, P. (2014). La comunidad de macroinvertebrados bentónicos de la cuenca superior del río Gallegos (Santa Cruz) y su relación con variables ambientales. Congreso Argentino de Limnología. La Plata.

ASUETA, R., VALLEJOS, J., MARTIN, J., FRANCISCOVIC, K., GUGLIELMINETTI, G., y AGUAS, P. (2013). Macroinvertebrados Bentónicos de la cuenca del río Gallegos, Santa Cruz. Su asociación con la Calidad Ambiental. $4^{\circ}$ Congreso de Ciencias Ambientales (COPIME) . Buenos Aires.

ASUETA, R., VALLEJOS, J., MARTIN, J., GUGLIELMINETTI, G., y AGUAS, P. (2014). Aplicación de índices bióticos basados en macroinvertebrados bentónicos en la cuenca superior del río Gallegos (Patagonia Austral, Argentina) . XXVI Reunión Anual de Ecología. Comodoro Rivadavia.

BONADA, N., PRAT, N., RESH, V., y STATZNER, B. (2006). Developments in aquatic insect biomonitoring: a comparative analysis of recent approaches. Entomology, 495523. https://doi.org/10.1146/annurev.ento.51.110104.151124

CRANSTON, P. (1995). The Chironomidae. The biology and ecology of nonbiting midges. En P. Armitage, P. Cranston, \& L. Pinder, Biogeography (págs. 62-84). London : Chapman \& Hall. https://doi.org/10.1007/978-94-011-0715-0_4

CRETTAZ-MinAGliA, M., JUÁREZ, R., AGUER, I., BORRO, E., y PERUZZO, R. (2014). Aplicación de índices de calidad de agua en un arroyo pampeano utilizando macroinvertebrados bentónicos como bioindicadores (Gualeguaychú, Entre Ríos, Argentina). Biología Acuática.

DOMINGUEZ, E., y FERNANDEZ, H. (2009). Macroinvertebrados bentónicos sudamericanos. Sistemática y biología. Tucumán: Fundación Miguel Lillo.

DONATO, M., MASSAFERRO, J., y BROOKS, S. (2009). Estado de conocimiento taxonómico de la fauna de Chironomidae (Diptera: Nematocera) de la Patagonia . Sociedad Entomológica Argentina, 187-192.

EPELE, L., MISERENDINO, M. L., y BRAND, C. (2012). Epele, L. B., Miserendino,Does nature and persistence of substrate at a mesohabitat scale matter for Chironomidae assemblages? A study of two perennial mountain streams in Patagonia, Argentina. Journal of Insect science, 68. https://doi.org/10.1673/031.012.6801

HANSON, P., SPRINGER, M., y RAMIREZ, A. (2010). Introducción a los grupos de macroinvertebrados acuáticos. Biología Tropical, 3-37. https://doi.org/10.15517/rbt.v58i4.20080

HELAWELL, J. (1986). Biological indicators of Freshwater Pollution and Environmental Management. Elsevier applied Science. https://doi.org/10.1007/978-94-009-4315-5

MAUAD, M. (2014). Tesis Doctoral. Comparación y aplicabilidad de índices bióticos para evaluar la calidad de aguas en ambientes lóticos del Parque Nacional Nahuel Huapi. Obtenido de Repositorio Institucional de la UNLP: http://sedici.unlp.edu.ar/handle/10915/34837

MEDINA, A., y PAGGI, A. (2004). Composición y abundancia de Chironomidae (Diptera) en un río serrano de zona semiárida (San Luis, Argentina). Sociedad Entomológica Argentina, 107-118. 
MISERENDINO, M. L. (2001). Macroinvertebrate assemblages in Andean Patagonian rivers and streams: environmental relationships. Hydrobiologia, 147-158. https://doi.org/10.1023/A:1017519216789

PAGGI, A. (1999). Los Chironomidae como indocadores de calidad de ambientes dulceacuícolas. Sociedad Entomológica Argentina, 202-207.

PAGGI, A. (2009). Diptera: Chironomidae. En E. Dominguez, \& H. Fernandez, Macroinvertebrados bentónicos sudamericanos. Sistemática y Biología. Tucumán : Fundación Miguel Lillo.

PAVE, P., y MARCHESE, M. (2005). Pave, PInvertebrados bentónicos como indicadores de calidad del agua en ríos urbanos (Paraná-Entre Ríos, Argentina). Ecología Austral, 183-197.

PRAT, N., RÍOS, B., ACOSTA, R., y RIERADEVALL, M. (2009). Los macroinvertebrados como indicadores de calidad de las aguas. Sistemática y biología, 631-654.

ROLDÁN-PÉREZ, G. (2016). Los macroinvertebrados como bioindicadores de la calidad del agua: cuatro décadas de desarrollo en Colombia y Latinoamerica. Academia Colombiana de Ciencias Exactas, Físicas y Naturales, 254-274. https://doi.org/10.18257/raccefyn.335

TORRENTES, M., QUINTERO, O., ATILANO, E., VARGAS, H., MESA, J., CORTÉS, F., y RAMÍREZ, N. (2016). Aplicación de tres índices bióticos en el río San Juan, Andes, Colombia. Mutis, 59-73. https://doi.org/10.21789/22561498.1151

ZANOTTO-ARPELliNO, J., PRÍNCIPE, R., OBERTO, A., y GUALDONI, C. (2015). Variación espacio-temporal de Chironomidae (Diptera) bentónicos y derivantes en un arroyo serrano en Córdoba, Argentina. Iheringia, 41-52.

https://doi.org/10.1590/1678-4766201510514152 